> Les avancées en recherches génétique et génomique ne cessent d'accroître nos connaissances des maladies héréditaires. Un nombre croissant de ces maladies relève d'épissages aberrants qui représentent des cibles idéales pour les approches correctives centrées sur l'ARN. De nouvelles stratégies, en particulier médicamenteuses, visant à exclure ou à ré-inclure des exons lors du processus d'épissage, ont ainsi émergé et plusieurs molécules ont récemment obtenu des autorisations de mise sur le marché, notamment pour le traitement de la dystrophie musculaire de Duchenne et de l'amyotrophie spinale, suscitant de plus en plus d'intérêt et d'espoir. Parmi ces molécules, les oligonucléotides antisens, ou ASO, ont connu un réel essor et font l'objet de progrès constants en matière de modifications chimiques et de conception. Toutefois, leur biodistribution après administration par voie générale demeure souvent limitée, et le développement de chimies alternatives plus performantes et de nouveaux systèmes d'adressage est devenu un axe de recherche très actif. En parallèle, l'utilisation de petites molécules présentant une excellente biodistribution, ou de vecteurs viraux pour véhiculer les séquences antisens, est également explorée. Dans cette Synthèse, nous présentons les dernières avancées de ces approches de modulation d'épissage à travers deux exemples de maladies neuromusculaires. Nous discutons de leurs avantages et des principales limitations actuelles. <

Les stratégies thérapeutiques fondées sur la modulation de l'épissage de l'ARN messager (ARNm) sont actuellement en pleine expansion, notamment grâce à de récents succès dans le traitement de certaines maladies neuromusculaires. Au cours de la dernière

Vignette (Photo @ Theresinha Evangelista).

\section{Les approches thérapeutiques de modulation de l'épissage}

\section{Avancées et perspectives}

Amel Saoudi ${ }^{1,2}$, Aurélie Goyenvalle ${ }^{1,3}$

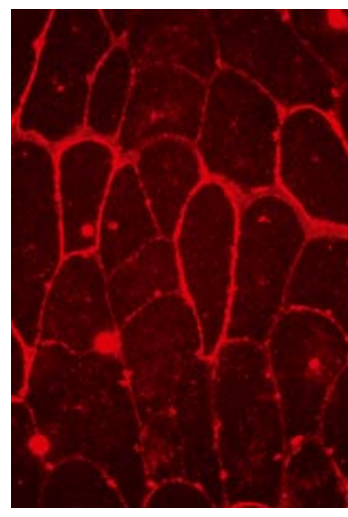

${ }^{1}$ Université Paris-Saclay, UVSQ, Inserm, END-ICAP, Handicap neuromusculaire physiopathologie, biothérapie et pharmacologie appliquées, 78000 Versailles, France. ${ }^{2}$ Université Paris-Saclay, CNRS, Institut des neurosciences Paris Saclay, 91190 Gif-sur-Yvette, France.

${ }^{3}$ Laboratoire international associé Biothérapies appliquées aux handicaps neuromusculaires (LIA BAHN), Centre scientifique de Monaco, Monaco.

décennie, plusieurs molécules ont obtenu des autorisations de mise sur aurelie.goyenvalle@uvsq.fr Duchenne (DMD) et de l'amyotrophie spinale (SMA), et de nouvelles générations de molécules sont désormais en cours de développement. La possibilité d'agir sur l'épissage des ARN pré-messagers (pré-ARNm) présente un intérêt majeur pour de nombreuses maladies génétiques causées par des mutations affectant l'épissage. La modulation de I'ARN repose sur l'utilisation de divers outils moléculaires, tels que des petites molécules actives, au premier rang desquelles les oligonucléotides antisens (ASO), qui peuvent être administrés directement ou véhiculés par des vecteurs viraux. Les ASO sont des oligonucléotides synthétiques de 12 à 30 nucléotides conçus pour se lier à un ARNm ou à un ARN non codant par appariement de bases Watson-Crick (guanine-cytosine et adénine-uracile), dans le but de moduler l'expression de ces ARN. Les ASO ont été utilisés pour la première fois en 1978 par Zamecnik et Stephenson afin d'inactiver l'expression des gènes [1], mais la technique a rapidement évolué, notamment grâce aux progrès faits en termes de modifications chimiques, ce qui a permis d'élargir considérablement les champs d'applications possibles. Alors que les premiers ASO, de type phosphodiester ( $P O$ ) non modifiés, étaient rapidement dégradés dans la circulation sanguine, diverses modifications ont progressivement été introduites dans leur squelette/structure chimique afin d'augmenter leur stabilité et leur affinité pour l'ARN [2]. L'une des premières modifications a été le remplacement d'un des atomes d'oxygène du groupement phosphate $\mathrm{PO}_{4}$ par un atome de soufre, créant ainsi des liens phosphorothioate (ou thiophosphate) (PS). Cette substitution a non seulement permis d'améliorer la résis- 
tance endogène des ASO aux nucléases, mais a aussi offert un avantage significatif en termes de pharmacocinétique. En effet, les ASO-PS (ASO de type phosphorothioate) présentent une affinité accrue pour de nombreuses protéines $[3,4]$, ce qui facilite leur distribution et leur absorption cellulaires par rapport à leurs homologues phosphodiester. Cependant, les modifications de type PS sont également connues pour être à l'origine d'effets indésirables dus à leur affinité pour certaines protéines plasmatiques, conférant parfois aux ASO-PS un profil toxicologique indésirable [5]. Afin d'améliorer leur résistance aux nucléases et leur affinité pour les ARNm ciblés, des ASO de deuxième génération ont été alors développés, avec l'introduction de modifications chimiques en position 2' du ribose, telles que les 2'0-méthyl (2'0Me) et 2'0-méthoxyéthyl (2'MOE) [6], ou par des changements substantiels dans la structure du sucre, conduisant à une grande variété de molécules, comme les morpholinos (ou PMO pour phosphoroamidate morpholino oligomer) [7] et les oligonucléotides de type contraint (LNA pour locked nucleic acid [8] et cEt pour 2'-4'-constrained ethyl [9]).

Ces avancées en matière de modifications chimiques ont incontestablement contribué au succès de certains ASO. Le domaine de la modulation d'épissage a cependant connu plusieurs tentatives infructueuses, mettant en évidence les limites des outils actuels.

Nous proposons ici de faire le point sur l'état actuel des thérapies fondées sur la modulation d'épissage et de mettre en perspectives leurs progrès récents à travers les développements précliniques et cliniques réalisés pour deux maladies : la dystrophie musculaire de Duchenne et l'amyotrophie spinale.

\section{Le saut d'exon thérapeutique pour la dystrophie musculaire de Duchenne}

La première approche de modulation de l'épissage à avoir été évaluée en clinique fut le saut d'exon thérapeutique développé pour traiter la dystrophie musculaire de Duchenne (DMD). La DMD est une maladie neuromusculaire sévère liée au chromosome $X$ et affectant 1 naissance sur 5000 naissances d'enfants de sexe masculin. Elle se caractérise par une faiblesse musculaire progressive et une dégénérescence des muscles, dues à des mutations dans le gène $D M D$, qui modifient le cadre de lecture et empêchent la production de la dystrophine, une protéine qui joue un rôle mécanique et signalétique entre le cytosquelette d'actine et la matrice extracellulaire. L'approche du saut d'exon thérapeutique pour la DMD vise à éliminer un exon lors de l'épissage, en utilisant des ASO masquant les sites d'épissage, afin de restaurer le cadre de lecture originel, ce qui permet la production d'une dystrophine, parfois nommée quasi-dystrophine, qui, bien que légèrement tronquée, reste fonctionnelle (Figure 1). Plusieurs chimies d'ASO ont été développées pour la DMD dans le but d'augmenter la stabilité des ASO et leur affinité pour l'ARN cible. Parmi celles-là, le 2'OMe chargé (ou drisapersen) et le PMO non chargé (ou étéplirsen) ont été les premiers ASO utilisés pour cibler l'exon 51 du gène DMD. Malgré des résultats encourageants dans les études cliniques initiales [10], un grand essai de phase III évaluant l'administration par voie sous-cutanée du drisapersen n'a pas démontré d'avantages cliniques significatifs ni d'augmentation évidente de la dystrophine [11]. Ces résultats décevants, ainsi que l'observation de certains effets secondaires, tels qu'une protéinurie et des réactions au niveau du site d'injection, ont conduit la Food and Drugs Administration (FDA) des États-Unis à rejeter le drisapersen, conduisant ainsi à l'arrêt de tous les programmes cliniques utilisant des 2'OMe pour la DMD, y compris ceux ciblant d'autres exons (les exons 45, 44 et 53 ).

En revanche, l'injection du PMO (étéplirsen) par voie intraveineuse a conduit à des niveaux de production faibles mais significatifs de la dystrophine, ce qui lui a permis d'obtenir en décembre 2016, non sans controverse, une autorisation de mise sur le marché de la FDA, l'étéplirsen devenant ainsi le premier ASO modulateur d'épissage à être approuvé aux États-Unis [12]. D'autres ASO de type PMO, ciblant I'exon 53 ont, par la suite, été approuvés par la FDA en décembre 2019 (golodirsen) et en août 2020 (viltolarsen) sur la base d'une faible restauration de dystrophine (en moyenne $1 \%$ pour le golodirsen et $2,8 \%$ pour le viltolarsen chez les patients traités par $80 \mathrm{mg} / \mathrm{kg}$ de PMO) [13, 14]. Un autre PMO ciblant l'exon 45 (le casimersen) vient également d'être approuvé par la FDA en février 2021 sur la base d'une restauration de dystrophine de $1,7 \%^{1}$ chez les patients traités par $30 \mathrm{mg} / \mathrm{kg}$ de PMO. En dépit de ces apparents succès, les niveaux de dystrophine restauré dans les biopsies de patients traités par ces PMO restent extrêmement faibles et, en 2018, l'Agence européenne des médicaments (AEM) a émis un avis négatif concernant l'étéplirsen [12], jugeant que le rapport bénéfice-risque actuel n'était pas positif et que l'utilisation de la dystrophine comme biomarqueur était encore prématurée. Afin de fournir des preuves concluantes de l'efficacité clinique de ces molécules, de vastes études de phase III contrôlées contre placebo sont en cours avec l'étéplirsen, le golodirsen et le casimersen.

L'une des limitations principales des ASO de première génération demeure leur adressage médiocre aux tissus cibles, comme les muscles squelettiques ou le cœur. II a notamment été montré que l'absorption musculaire des PMO dépendait de la présence de foyers inflammatoires associés aux lésions dystrophiques, ainsi que de la fusion des myoblastes et des monocytes chargés en PMO au voisinage des fibres musculaires endommagées [15]. L'amélioration de l'adressage des ASO à leurs tissus cibles représente aujourd'hui un défi majeur dans

Résultats non publiés communiqués par Sarepta : https://investorrelations. sarepta.com/news-releases/news-release-details/sarepta-therapeuticsannounces-positive-expression-results. 


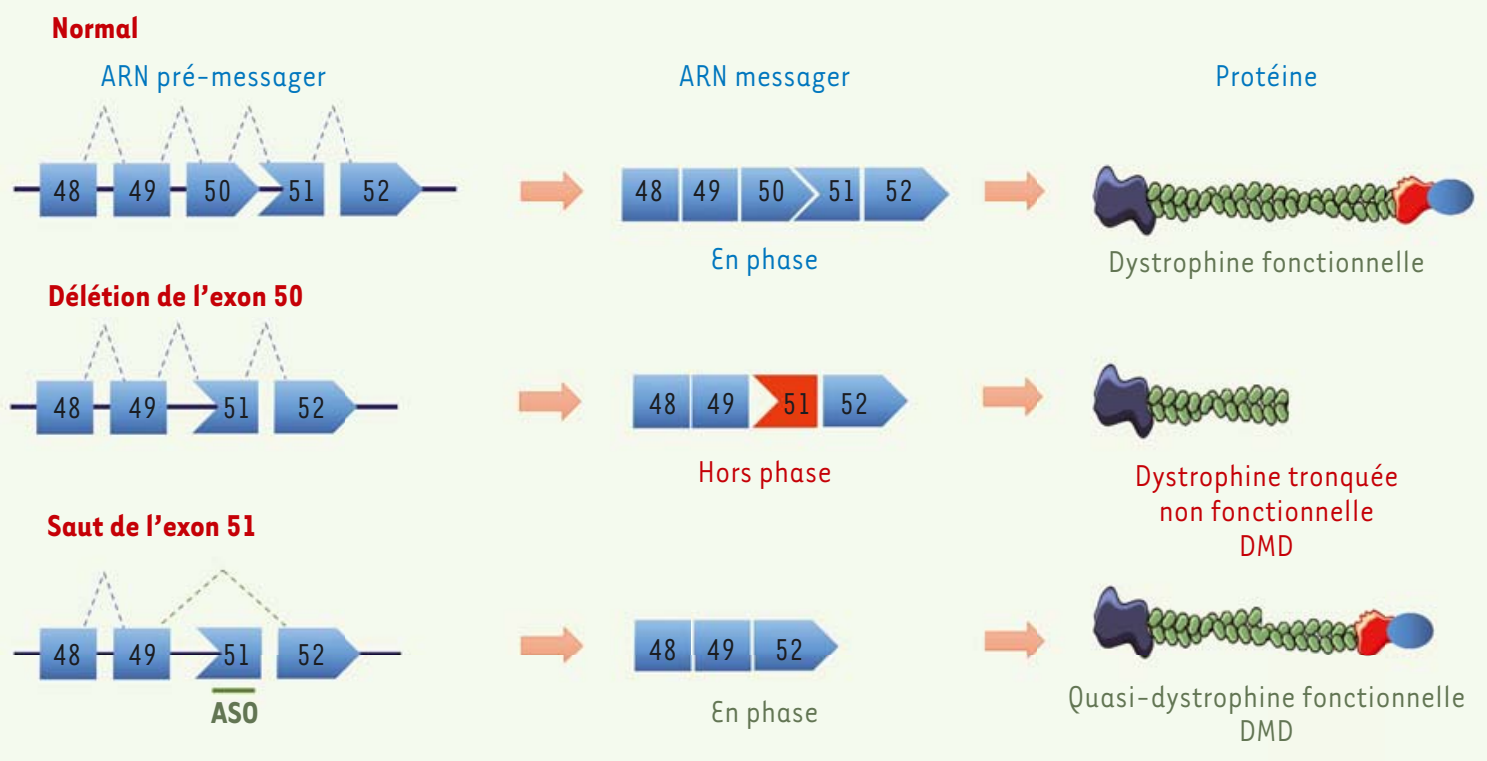

Figure 1. Principe du saut d'exon thérapeutique pour le traitement de la DMD. L'utilisation d'ASO vise à masquer les sites clés d'épissage, afin d'exclure un exon (par exemple, ici, l'exon 51) pour restaurer le cadre de lecture de l'ARNm DMD, permettant ainsi la synthèse d'une protéine quasi-dystrophine, légèrement plus courte que la dystrophine, mais tout de même fonctionnelle (similaire aux quasi-dystrophines produites par les patients atteints de myopathie de Becker).

le domaine de la modulation d'épissage thérapeutique ; de nouvelles générations de molécules antisens visant à relever ce défi sont en cours de développement [16].

\section{Nouvelles générations d'ASO et alternatives à l'adressage}

Des ASO dits «stéréopurs » ont récemment été développés dans le but d'optimiser les propriétés de biodistribution des ASO présentant des liaisons PS ainsi que leur profil toxicologique. Étant donné que le squelette phosphorothioate est chiral, un ASO-PS de 20 nucléotides est en fait un mélange de $2^{19}$ stéréoisomères différents qui ne sont pas tous aussi efficaces, ni bien tolérés. Un ASO «stéréopur 》 a été ainsi sélectionné en fonction de la chiralité $\mathrm{R}$ ou $\mathrm{S}$ de chaque phosphate.

Un candidat «stéréopur 》 optimisé pour le saut de l'exon 51 (suvodirsen) a ainsi démontré une restauration de dystrophine bien supérieure (environ $52 \%$ de restauration versus $1 \%$ obtenu avec les autres types d'ASO) dans des cellules isolées de patients présentant une DMD. Cependant, son évaluation clinique en phase II/III (NCT03907072) n'a montré aucune induction de l'expression de la dystrophine après des administrations intraveineuses, et le développement clinique du suvodirsen pour la DMD a été suspendu.

La recherche de chimies alternatives d'ASO a conduit à l'identification des tricyclo-ADN (tcDNA) comme des ASO prometteurs pour les approches de modulation d'épissage thérapeutiques [17, 18]. Les tricyclo-ADN sont des analogues synthétiques de conformation contrainte, ayant notamment démontré des propriétés de biodistribution intéressantes, y compris une capacité à franchir la barrière hémato-encéphalique après une administration par voie générale [19] $(\rightarrow)$.

$(\rightarrow)$ Voir la Nouvelle de A.Goyenvalle et al., $\mathrm{m} / \mathrm{s} \mathrm{n}^{\circ} \mathrm{3}$, mars 2015 , page 253
Cet avantage ouvre des perspectives uniques de pouvoir traiter à la fois la maladie musculaire et les comorbidités de la DMD dues à l'absence de dystrophine dans le cerveau. Un essai clinique de phase I/II est programmé en France pour fin 2021 par la société SPy Therapeutics.

La conjugaison des ASO à des peptides facilitant leur pénétration dans les cellules (appelés CPP pour cellpenetrating peptide) ou à des anticorps, tels que ceux ciblant le récepteur de la transferrine [20], est également étudiée pour améliorer une administration ciblée. Les CPP sont de courts peptides cationiques et/ou amphipathiques, qui facilitent la translocation des ASO à travers les membranes cellulaires ainsi que leur sortie des endosomes. Certains de ces ASO conjugués à de tels peptides, tels que les PPMO (peptide-conjugated phophoroamidate morpholino oligomer) ont démontré une efficacité très supérieure à leurs homologues non conjugués, permettant notamment une restauration significative de la production de dystrophine dans le cœur de souris modèles de la maladie [21]. Une étude de phase I/II est actuellement en cours avec un PPMO ciblant l'exon 51, et Sarepta Therapeutics a récemment communiqué des résultats préliminaires encourageants, notamment en comparaison de patients traités par l'étéplirsen (le PMO équivalent, mais non conjugué à un peptide). 
Afin de pérenniser l'effet des ASO, et ainsi d'éviter la nécessité d'une administration récurrente (potentiellement à vie), il a été proposé de vectoriser les séquences antisens dans des vecteurs recombinants dérivés des virus adéno-associés (AAV). Pour cela, les séquences antisens sont insérées dans un ARN U7snRNA (U7 small-nuclear RNA) optimisé. L'ARN U7snRNA est un petit ARN nucléaire non codant qui est naturellement impliqué dans la maturation des pré-ARNm des histones. Pour en faire un outil de modulation d'épissage, la séquence antisens naturelle du U7snRNA est remplacée par une séquence antisens d'intérêt (ciblant par exemple un exon du gène DMD). Grâce à cette inclusion dans I'ARN U7snRNA, et sa vectorisation dans un vecteur de type AAV, la séquence antisens est directement adressée et exprimée dans le noyau, de façon continue sous le contrôle du promoteur naturel de l'ARN U7snRNA. Des études précliniques, réalisées dans des modèles murins de $\operatorname{DMD}[19,22]$ et chez le chien Golden retriever muscular dystrophy (GRMD) ${ }^{2}[23,24]$, ont montré que cette approche permet une localisation subcellulaire appropriée des séquences antisens avec les pré-ARNm, grâce à l'accumulation des particules U7snRNP dans le noyau [25], ainsi qu'une restauration de l'expression de dystrophine jusqu'à cinq ans post-injection chez le chien GRMD. À la suite de ces résultats encourageants, un essai clinique de phase I/II (NCT04240314) pour le saut de l'exon 2 a été entrepris chez des patients atteints de DMD et présentant une duplication de cet exon. Les duplications d'exon représentent en effet $11 \%$ des mutations touchant le gène $D M D$, et la duplication de l'exon 2 est la plus commune chez les patients [26]. Le saut d'exon ciblant les duplications vise à restaurer une dystrophine complète et donc entièrement fonctionnelle, ce qui présente un avantage significatif par rapport aux quasi-dystrophines résultant de l'élimination d'exon(s) interne(s) pour corriger le cadre de lecture ou les mutations non-sens. Les résultats préliminaires de cette étude de phase I/ II ont été présentés lors du $25^{\mathrm{e}}$ congrès de la World Muscle Society (WMS) en octobre 2020 [27] et ont révélé, chez les 2 patients traités, une diminution de respectivement $95 \%$ et $81 \%$ des niveaux de créatine kinase sérique (typiquement élevés chez les patients atteints de DMD) à trois mois postinjection, et une réexpression respective de $5,8 \%$ et $1,4 \%$ de dystrophine, confirmant le potentiel thérapeutique de cette approche vectorisée.

L'ensemble des données désormais disponibles sur les thérapies de modulation d'épissage pour la DMD suggère que l'adressage ciblé des ASO médicaments représente la limitation majeure qui entrave leur efficacité lorsqu'ils sont administrés par voie générale. En revanche, pour d'autres maladies neuromusculaires, telles que la SMA, pour laquelle les motoneurones sont les principales cellules cibles, ce problème d'adressage a pu être contourné en utilisant une voie d'administration ciblée et a ainsi conduit à des succès beaucoup plus marquants.

\section{La modulation d'épissage pour l'amyotrophie spinale}

L'amyotrophie spinale (SMA) est une maladie autosomique récessive du motoneurone avec une incidence de 1/10 000 naissances. Elle est

${ }^{2}$ Ce modèle de chien atteint de myopathie de Duchenne présente spontanément une anomalie dans le gène $D M D$; il s'agit d'une mutation entraînant la perte de l'exon 7 du gène. causée par des mutations dans le gène SMN1 (survival motor neuron 1) codant la protéine SMN, impliquée dans la maturation des ARNm via l'assemblage du spliceosome. Les patients atteints de SMA sont classés en 4 groupes (type 1 à 4) selon l'âge de début de la maladie et l'importance du déficit de la force musculaire, mais la variante la plus commune est la SMA de type 1 , qui représente $60 \%$ des cas. Elle affecte les nourrissons, qui n'acquièrent jamais la capacité de s'asseoir et survivent rarement au-delà de l'âge de 2 ans. II existe une copie centromérique du gène, appelée $S M N 2$, qui ne diffère du gène SMNI que par quelques nucléotides, dont une substitution $C->T$ qui entraîne un épissage alternatif de l'exon 7 et qui ne permet la production que de $10 \%$ de protéine SMN, une production insuffisante pour contrebalancer le manque de SMN et maintenir la survie et le fonctionnement normal des motoneurones. Diverses thérapies incluant des ASO et des petites molécules ont été mises au point pour moduler l'épissage du gène SMN2 et permettre la ré-inclusion de cet exon 7 afin de produire davantage de protéine SMN (Figure 2).

\section{Les ASO pour l'amyotrophie spinale}

Plusieurs classes d'ASO ont été développées pour interférer avec l'épissage de l'ARNm SMN2, parmi lesquelles un ASO de type 2'MOE-PS de 18 nucléotides, nommé nusinersen (commercialisé sous le nom de Spinraza ${ }^{\circledR}$ ). Celui-ci a démontré des résultats particulièrement prometteurs, initialement dans les modèles murins de SMA [28-30], puis dans les études cliniques. Tout comme les autres ASO utilisés actuellement en clinique, le nusinersen ne traverse pas la barrière hématoencéphalique et nécessite des injections intrathécales directes, administrées sous la forme d'une phase de chargement (4 administrations sur une période de deux mois), suivie d'injections trimestrielles. Plusieurs essais cliniques randomisés ont été réalisés par lonis pharmaceuticals et Biogen, et ont permis de démontrer l'efficacité du nusinersen chez plusieurs centaines de patients atteints de SMA [31]

$(\rightarrow)$ Voir la Synthèse de $\varepsilon$. Gargaun, $m / s$ hors série $n^{\circ} 2$, novembre 2019, page 11

$(\rightarrow)$.

Dans l'étude multicentrique ENDEAR de phase III, en double aveugle, la survie et l'acquisition de nouvelles capacités psychomotrices chez des patients SMA de type 1 ont notamment conduit à l'interruption prématurée de l'étude contrôlée contre placebo, permettant ainsi à tous les participants de recevoir le médicament. Le nusinersen a été approuvé par la FDA fin décembre 2016 et par l'AEM en juin 2017. II représente probablement le plus grand succès de la thérapie à base d'ASO. Toutefois, plusieurs défis demeurent et, bien que le nusi- 


\section{SMN1}
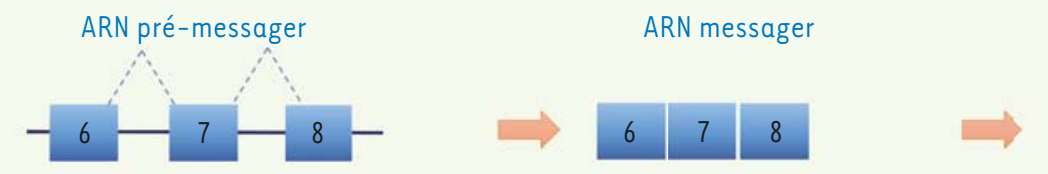

Protéine

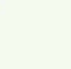

\section{SMN2}
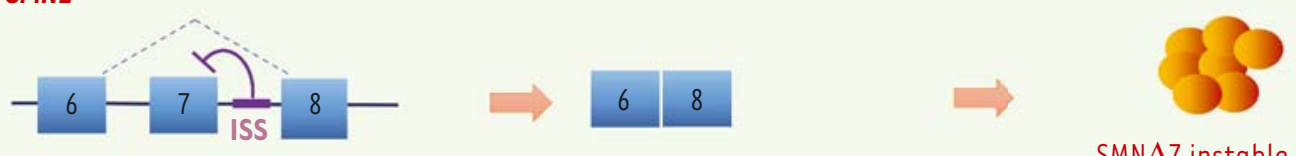

SMN $\Delta 7$ instable

SMN2 : Ré-inclusion de l'exon 7
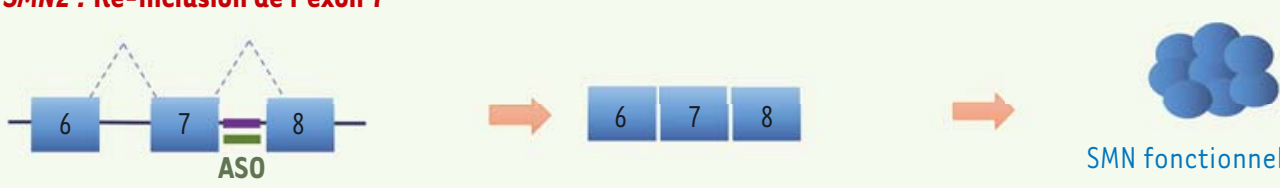

SMN fonctionnelle

Figure 2. Modulation de l'épissage de SMN2 pour le traitement de l'amyotrophie spinale (SMA). L'utilisation d'ASO ou de petites molécules dans le contexte de la SMA vise à renforcer l'inclusion de l'exon 7 dans l'ARNm SMN2 afin de produire une protéine SMN fonctionnelle. ISS : motif intronique inhibiteur d'épissage (Intronic splicing silencer).

nersen induise une amélioration clinique robuste et significative chez les patients atteints de SMA de type 1 (mesurée par une réponse positive significative du test HINE [Hammersmith Infant Neurological Examination ]), de nombreux enfants conservent un handicap résiduel significatif en raison du stade avancé de la maladie avant le début du traitement. Dans ce cadre, les résultats d'une étude de phase II en cours chez des nourrissons pré-symptomatiques susceptibles de développer une SMA de type 1 ou 2, a fourni des données extrêmement encourageantes sur l'efficacité d'un traitement pré-symptomatique, puisque $88 \%$ des patients traités sont parvenus à marcher de manière indépendante [32]. Au vu de ces résultats exceptionnels, le dépistage néonatal de la maladie a commencé dans de nombreux pays.

Néanmoins, le mode d'administration actuel par injections intrathécales ne permet pas de traiter l'atteinte d'organes périphériques rapportée dans la SMA, telle que les dysfonctionnements cardiaques, et représente également un risque de lésions tissulaires ou d'infection après de multiples administrations. Des chimies alternatives d'ASO telles que les PPMO [33] ou les tcDNA [18] ont démontré leur capacité à franchir la barrière hémato-encéphalique après administration par voie générale, notamment dans des modèles de souris SMA de type 3 , permettant d'envisager un bénéfice thérapeutique suite à des injections intraveineuses plutôt qu'intrathécales. Au-delà de ces nouvelles générations d'ASO, de petites molécules très prometteuses ont également été développées pour moduler l'ARNm de SMN2.

\section{Les petites molécules pour l'amyotrophie spinale}

Le risdiplam est une petite molécule issue de la recherche du laboratoire PTC Therapeutics et développée par le laboratoire Roche/ Genentech, permettant de moduler l'épissage de l'exon 7 du gène
SMN2 et ainsi de favoriser son inclusion dans l'ARNm de SMN2, via le renforcement de l'interaction entre le spliceosome (ou épissosome) et le site d'épissage 5' (5'SS) de l'exon 7 de SMN2 [34]. Le ridisplam permet en effet de stabiliser une adénine non hybridée au niveau de la jonction exon-intron, transformant ainsi le 5'SS « faible » de l'exon 7 en un site d'épissage nettement plus fort. Ce type de composés étant capable de traverser la barrière hémato-encéphalique avec une excellente biodistribution [35], le risdiplam peut être administré par voie orale, ce qui présente un avantage indiscutable pour les patients. Les résultats préliminaires de deux essais internationaux de phase II/III (I'essai FIREFISH dans la SMA de type 1 et l'essai SUNFISH randomisé, en double aveugle contre placebo, dans la SMA de type 2 ou 3) ont confirmé l'efficacité du risdiplam sur les fonctions motrices et respiratoires des patients. Sur la base de ces résultats, la FDA a accordé une AMM au risdiplam le 7 août 2020 pour les SMA de type 1, 2 et 3 aux États-Unis. II est commercialisé sous le nom de Evrysdi ${ }^{\circledR}$. En Europe, Roche a déposé une demande d'AMM en août 2020 à l'AEM qui a accepté une évaluation accélérée, ce qui signifie que le processus d'évaluation peut être ramené de 15 à 9 mois. Des essais sont actuellement toujours en cours pour évaluer ce médicament sur un grand groupe de patients atteints de SMA, représentatifs de la population concernée par cette maladie : les essais FIREFISH et SUNFISH se poursuivent; l'essai JEWELFISH est en cours chez des 
patients âgés de 6 mois à 60 ans, ayant déjà été traités par d'autres molécules, et l'essai RAINBOWFISH est en cours chez des nourrissons âgés de moins de 6 semaines et pré-symptomatiques.

Une autre molécule modifiant la maturation de l'ARNm SMN2, le branaplam, a été identifiée par Novartis Pharmaceuticals lors d'un crible à haut débit. Elle permet de stabiliser l'interaction entre l'épissosome et le pré-ARNm SMN2 [36]. En raison des résultats encourageants obtenus dans un modèle sévère de souris SMA, démontrant une augmentation significative de l'expression de la protéine SMN et de la survie des animaux, le branaplam est actuellement en cours d'évaluation clinique chez des patients SMA de type I (NCT02268552).

\section{Conclusions et perspectives}

Les thérapies par modulation d'ARN ont fait des progrès impressionnants au cours des dernières années, comme l'illustrent les récentes autorisations de mise sur le marché de plusieurs médicaments, et le nombre croissant d'essais cliniques évaluant ce type de stratégie. Ces succès récents suscitent optimisme et espoir pour le développement de futures thérapies de modulation d'épissage. Cependant, le bénéfice clinique de ces approches dépend de façon critique du tissu ciblé et de la voie d'administration, notamment dans le cas des oligonucléotides antisens (ASO). En effet, le potentiel thérapeutique des ASO administrés par voie générale est bien loin de celui obtenu avec des ASO injectés localement, comme en témoigne le succès du nusinersen, administré par voie intrathécale, comparé à l'effet beaucoup plus mitigé induit par l'étéplirsen pour la DMD, qui n'a d'ailleurs toujours pas reçu d'AMM en Europe. Cela s'explique par la biodistribution extrêmement limitée des ASO injectés par voie générale : il est estimé que seulement $1 \%$ environ des ASO atteint le compartiment cellulaire ciblé (comme les muscles squelettiques ou cardiaque) après une administration par voies intraveineuse ou sous-cutanée [37].

L'amélioration de la distribution ciblée (en particulier au système nerveux central ou au muscle cardiaque, dans le cas des maladies neuromusculaires) représente aujourd'hui le principal défi identifié dans le domaine de la modulation d'épissage induite par les ASO. Le développement de chimies alternatives plus performantes ou de nouveaux systèmes d'administration est devenu un axe de recherche très actif ces dernières années [16]. Le développement récent et la commercialisation d'ASO incorporant des conjugués GalNAc ( $\mathrm{N}$-acétylgalactosamine), présentant une supériorité thérapeutique de 20 à 30 fois, ont confirmé la possibilité de créer de meilleurs ASO. Inspirée par le succès des conjugués GalNAc, la conjugaison d'ASO à divers ligands, tels que des peptides, des lipides et des anticorps, est actuellement explorée [38], et ouvre la voie vers de futures thérapies toujours plus innovantes. $\diamond$

\section{SUMMARY}

RNA splicing modulation: Therapeutic progress and perspectives

Advances in genetic and genomic research continue to increase our knowledge of hereditary diseases, and an increasing number of them are being attributed to aberrant splicing, thus representing ideal targets for RNA modulation therapies. New strategies to skip or re-include exons during the splicing process have emerged and are now widely evaluated in the clinic. Several drugs have recently been approved in particular for the treatment of Duchenne muscular dystrophy and spinal muscular atrophy. Among these molecules, antisense oligonucleotides, or ASOs, have gained increasing interest and have constantly been improved over the years through chemical modifications and design. However, their limited biodistribution following systemic administration still represents a major hurdle and the development of more potent alternative chemistries or new delivery systems has become a very active line of research in the past few years. In parallel, the use of small molecules with excellent biodistribution properties or of viral vectors to convey antisense sequences is also being investigated. In this review, we summarize the recent advances in splicing therapies through two examples of neuromuscular diseases and we discuss their main benefits and current limitations. $\diamond$

\section{LIENS D’INTÉRÊT}

Les auteurs déclarent n'avoir aucun lien d'intérêt concernant les données publiées dans cet article.

\section{RÉFÉRENCES}

1. Zamecnik PC, Stephenson ML. Inhibition of Rous sarcoma virus replication and cell transformation by a specific oligodeoxynucleotide. Proc Natl Acad Sci USA $1978 ; 75: 280-4$.

2. Wickstrom $\varepsilon$. Oligodeoxynucleotide stability in subcellular extracts and culture media. J Biochem Biophys Methods 1986; $13: 97-102$.

3. Gaus HJ, Gupta R, Chappell AE, et al. Characterization of the interactions of chemically-modified therapeutic nucleic acids with plasma proteins using a fluorescence polarization assay. Nucleic Acids Res $2019 ; 47$ : 1110-22.

4. Crooke ST, Wang $S$, Vickers TA, et al. Cellular uptake and trafficking of antisense oligonucleotides. Nat Biotechnol $2017 ; 35$ : 230-7.

5. Crooke ST, Baker BF, Witztum JL, et al. The effects of 2'-0-methoxyethyl containing antisense oligonucleotides on platelets in human clinical trials. Nucleic Acid Ther $2017 ; 27: 121-9$.

6. Goyenvalle A, Leumann C, Garcia L. Therapeutic Potential of Tricyclo-DNA antisense oligonucleotides. J Neuromuscul Dis 2016 ; 3 : 157-67.

7. Summerton J, Weller D. Morpholino antisense oligomers: design, preparation, and properties. Antisense Nucleic Acid Drug Dev 1997 ; 7 : 187-95.

8. Hagedorn PH, Persson R, Funder ED, et al. Locked nucleic acid: modality, diversity, and drug discovery. Drug Discov Today $2018 ; 23$ : 101-14.

9. Seth PP, Siwkowski A, Allerson CR, et al. Design, synthesis and evaluation of constrained methoxyethyl ( $c M O E)$ and constrained ethyl (cEt) nucleoside analogs. Nucleic Acids Symp Ser (0xf) 2008 ; 553-4.

10. Goemans NM, Tulinius M, Akker JT van den, et al. Systemic administration of PR0051 in Duchenne's muscular dystrophy. N Engl J Med 2011 ; 364 : 1513-22.

11. Goemans N, Mercuri $\varepsilon$, Belousova $\varepsilon$, et al. A randomized placebo-controlled phase 3 trial of an antisense oligonucleotide, drisapersen, in Duchenne muscular dystrophy. Neuromuscul Disord $2018 ; 28: 4-15$.

12. Aartsma-Rus A, Goemans N. A sequel to the Eteplirsen saga: eteplirsen is approved in the United States but was not approved in Europe. Nucleic Acid Ther 2019 ; 29 : 13-5.

13. Heo YA. Golodirsen: first approval. Drugs $2020 ; 80: 329-33$.

14. Komaki H, Takeshima Y, Matsumura T, et al. Viltolarsen in Japanese Duchenne muscular dystrophy patients: a phase 1/2 study. Ann Clin Transl Neurol 2020 ; 7 : 2393-408. 


\section{RÉFÉRENCES}

15. Novak JS, Hogarth MW, Boehler JF, et al. Myoblasts and macrophages are required for therapeutic morpholino antisense oligonucleotide delivery to dystrophic muscle. Nat Commun $2017 ; 8$ : 941.

16. Roberts TC, Langer R, Wood MJA. Advances in oligonucleotide drug delivery. Nat Rev Drug Discov $2020 ; 19: 673-94$

17. Goyenvalle A, Griffith G, Babbs A, et al. Functional correction in mouse models of muscular dystrophy using exon-skipping tricyclo-DNA oligomers. Nat Med $2015 ; 21: 270-5$.

18. Robin V, Griffith G, Carter J-PL, et al. Efficient SMN rescue following subcutaneous tricyclo-DNA antisense oligonucleotide treatment. Mol Ther Nucleic Acids $2017 ; 7$ : 81-9.

19. Goyenvalle A, Griffith G, Avril A, et al. Un nouvel outil pour le traitement de la myopathie de Duchenne : les tricyclo-ADN. Med Sci (Paris) 2015 ; 31 : 253-6.

20. Sugo T, Terada M, Oikawa T, et al. Development of antibody-siRNA conjugate targeted to cardiac and skeletal muscles. J Control Release $2016 ; 237: 1-13$.

21. Betts C, Saleh AF, Arzumanov AA, et al. Pip6-PMO, a new generation of peptide-oligonucleotide conjugates with improved cardiac exon skipping activity for DMD treatment. Mol Ther Nucleic Acids $2012 ; 1$ : e38.

22. Goyenvalle A, Babbs A, Wright J, et al. Rescue of severely affected dystrophin/utrophin-deficient mice through scAAV-U7snRNA-mediated exon skipping. Hum Mol Genet $2012 ; 21: 2559-71$.

23. Vulin A, Barthélémy I, Goyenvalle $A$, et al. Muscle Function recovery in golden retriever muscular dystrophy after AAV1-U7 exon skipping. Mol Ther $2012 ; 20: 2120-33$.

24. Le Guiner C, Montus M, Servais L, et al. Forelimb treatment in a large cohort of dystrophic dogs supports delivery of a recombinant AAV for exon skipping in Duchenne patients. Mol Ther 2014 ; 22 : 1923-35.

25. Grimm C, Stefanovic B, Schümperli D. The low abundance of U7 snRNA is partly determined by its Sm binding site. EMBO J 1993 ; 12 : 1229-38.

26. Wein N, Vulin A, Falzarano MS, et al. Translation from a DMD exon 5 IRES results in a functional dystrophin isoform that attenuates dystrophinopathy in humans and mice. Nat Med $2014 ; 20$ : 992-1000.

27. Waldrop M. Expression of apparent full-length dystrophin in skeletal muscle in a first-in-human gene therapy trial using the scAAV9.U7-ACCA vector. Neuromuscul Disord 2020 ; S166-7.

28. Singh NK, Singh NN, Androphy $\varepsilon$, et al. Splicing of a critical exon of human survival motor neuron is regulated by a unique silencer element located in the last intron. Mol Cell Biol $2006 ; 26: 1333-$ 46.
29. Hua Y, Sahashi K, Hung G, et al. Antisense correction of SMN2 splicing in the CNS rescues necrosis in a type III SMA mouse model. Genes Dev $2010 ; 24$ : 1634-44.

30. Hua Y, Sahashi K, Rigo F, et al. Peripheral SMN restoration is essential for long-term rescue of a severe SMA mouse model. Nature 2011; 478: 123-6.

31. Gargaun $\varepsilon$. Les oligonucléotides anti-sens dans la SMA : retour d'expérience et données de la littérature. Med Sci (Paris) 2019 ; 35 (hors série ${ }^{\circ}{ }^{2}$ 2) : 11-4.

32. De Vivo DC, Bertini $\varepsilon$, Swoboda KJ, et al. Nusinersen initiated in infants during the presymptomatic stage of spinal muscular atrophy: Interim efficacy and safety results from the Phase 2 NURTURE study. Neuromuscul Disord $2019 ; 29: 842-56$.

33. Hammond SM, Hazell G, Shabanpoor F, et al. Systemic peptide-mediated oligonucleotide therapy improves long-term survival in spinal muscular atrophy. Proc Natl Acad Sci USA 2016 ; 113 : 10962-7.

34. Campagne $S$, Boigner $S$, Rüdisser $S$, et al. Structural basis of a small molecule targeting RNA for a specific splicing correction. Nat Chem Biol $2019 ; 15: 1191-8$

35. Poirier A, Weetall M, Heinig K, et al. Risdiplam distributes and increases SMN protein in both the central nervous system and peripheral organs. Pharmacol Res Perspect $2018 ; 6$ : e00447.

36. Cheung AK, Hurley B, Kerrigan R, et al. Discovery of small molecule splicing modulators of survival motor neuron-2 (SMN2) for the treatment of spinal muscular atrophy (SMA). J Med Chem 2018; 61 : 11021-36.

37. Godfrey C, Desviat LR, Smedsrød B, et al. Delivery is key: lessons learnt from developing splice-switching antisense therapies. EMBO Mol Med 2017 ; 9 : 545-57.

38. Bizot F, Vulin A, Goyenvalle A. Current status of antisense oligonucleotidebased therapy in neuromuscular disorders. Drugs $2020 ; 80$ : 1397-415.

TIRÉS À PART

A.Goyenvalle

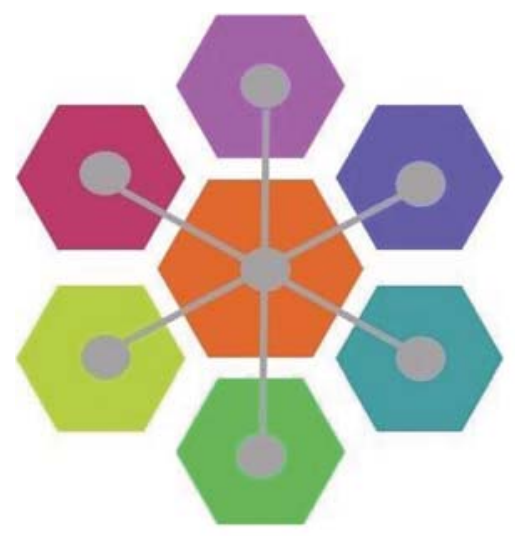

Global Registry for COL6-related dystrophies

\section{Registre global des dystrophies} liées au collagène de type VI

S'inscrire sur : www.collagen6.org

Ou contactez-nous par e-mail à l'adresse : collagenbregistry@ncl.ac.uk

\section{La traduction française sera bientôt disponible sur le site web.}

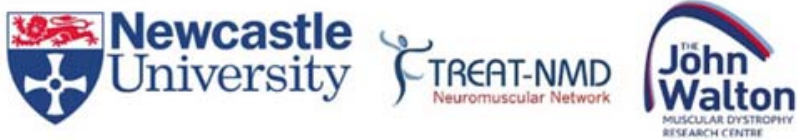

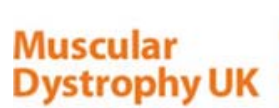

Muscular
Dystrophy UK

Fighting muscle-wasting conditions

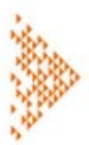

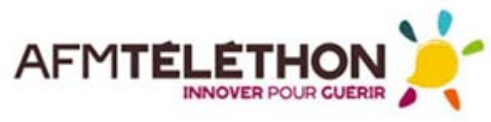

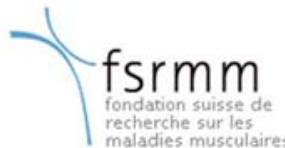

